\title{
Effect of different parities on reproductive performance, birth intervals, and tail behavior in sows
}

\author{
Ka Young Yang, Jung Hwan Jeon, Kyeong Seok Kwon, Hee Chul Choi, Jong Bok Kim and Jun Yeob Lee*
}

National Institute of Animal Science, Rural Development Administration, Wanju-gun 55365, Korea

\section{Abstract}

A goal for swine farming is the improvement in the number of live-born and weaned piglets per sow. Hence, the effect of parities should consider the correlation between the component traits of reproductive performance, weaning, and duration. Sows were housed in farrowing pens (W $2.2 \times \mathrm{D} 1.8 \times \mathrm{H} 1.2 \mathrm{~m}$ ) on a partially slatted plastic floor. Twenty sows used in this study were between the first and sixth parity in gilts (P1), parity 2-5 (P3), and parity 6-9 (P6). Data collection by parity was classified into three categories: (1) reproduction performance (gestation length, total number of piglets born, number of piglets live born, number of piglets stillborn, total piglet birth weight; (2) weaning traits (weaning period, number of piglets weaning, total piglets weaning weight); (3) duration traits (farrowing duration, placenta expulsion duration, time from last piglet to first placenta, average birth interval, and tail wagging behavior). Gestation length was higher in P6 than P1 and P3 of different parity sows. The maximum value in $\mathrm{P} 1$ and $\mathrm{P} 3$ was 117 days, but the median value in P6 was 117 days $(p<0.05)$. The total number of piglets born $(p=0.113)$, number of piglets live born $(p=0.118)$, number of still piglets born $(p=0.151)$, and total piglet birth weight $(p=0.117)$ were not affected by parity. The number of live piglets was higher than the other parities by an average of $15.6 \pm 2.1$ in P1. The duration of farrowing was the lowest at 22.2 min in P6, but the maximum value was 42.2 min more than other parities $(p=0.355)$. Weaning traits of sows also did not differ significantly $(p>0.05)$, but the weaning period from $\mathrm{P} 1$ was lower than that of the other parities $(p=0.170)$. The number of piglets weaned was 10 heads on average in P1, P3, and P6 ( $p<0.05)$. However, the mean values of the total piglet weaning weight $(p=0.377)$ of P6 (62.0 $10.4 \mathrm{~kg}$ ) were higher than those of P1 (54.9 $10.2 \mathrm{~kg})$ and P3 (58.4 $13.6 \mathrm{~kg})$. The placenta expulsion duration was higher in P6 than that in P1 and P3 ( $p=0.447)$. The time from the last piglet to first placenta was be lower in P3 than that of the other parities $(p=0.206)$. The average birth interval was higher in $\mathrm{P} 3$ than that of the other parities $(p=0.156)$. Tail wagging (count) behavior was higher in P6 than in the other parities $(p=0.065)$. The data showed that the reproduction performance, total piglets born, and weight were higher in the gilts group, and higher in the weaning trait than that in the P6 group. This study examined the relationship between reproductive performance, birth interval and tail motion according to sow parity. Regarding duration, farrowing duration was lower in $\mathrm{P} 6$ than that in the other parities, but placenta expulsion and tail wagging were higher in P6 than in the other parities. Therefore, it is possible that the results from these sows could be used as basic data for effective farm management.

Keywords: Birth intervals, Farrowing, Parity, Sows, Tail wagging

Received: Feb 272019 Revised: Apr 12,2019 Accepted: May 15, 2019

"Corresponding author: Jun Yeob Lee, National Institute of Animal Science, Rural Development Administration, Wanju-gun 55365, Korea.

Tel: +82-63-238-7408,E-mail: andrewlee@korea.kr

This is an Open Access article distributed under the terms of the Creative Commons Attribution Non-Commercial License (http://creativecommons.org/licenses/by$\mathrm{nc} / 4.0 /$ ) which permits unrestricted non-commercial use, distribution, and reproduction in any medium, provided the original work is properly cited.

Copyright (C) 2019 Korean Society of Animal Science and Technology. 


\section{Background}

Farrowing of sows in swine farms is closely related to economic efficiency. In addition to gilt, sows of different parity are required to have special help during farrowing from managers [1]. Further, the process of birth is a stressful event for the sow as well as for the piglets [2]. Good maternal ability in sows is characterized by high piglet growth rate and no piglet mortality [3]. The number of liveborn piglets and birth weight in sows are major reproductive and economic traits. For piglets, the birth process can be a struggle for life and death [2]. The majority of piglets die within the first 2-4 days of life [4]. Approximately 75\% of stillborn piglets die during parturition [3]. Several reports on the duration of farrowing (and/ or on birth intervals) have been published $[2,3,5,6]$. However, most of these reported results have suggested farrowing duration is related to the duration of farrowing parity, litter size, genotype management system. In addition, studies on the interval of piglets in gilts have been carried out $[7,8]$, but there has been no study on the interval of piglets in sows according to various parities.

Recently, animal welfare research has been conducted on livestock emotions [9,10]. Regarding pigs, various indexes of emotions such as tails, ears and facial expressions have being studied in various ways [11]. It has been suggested that tail movements in general could be a new potential behavioral indicator of positive emotions in gilts. However, reports about tail movement behavior relative to farrowing are limited. Sows are very limited in their behavior in farrowing crates, and the degree of stress varies with individuals [12]. When farrowing, gilts have been reported to exhibit abnormal behavior in farrowing crates [13]. These abnormal behaviors of sows may vary according to parity, but are more frequent in sows with higher parity than gilts [14]. Although studies on farrowing related to sow management (such as changes in posture, nursing, lying down behavior, etc.) have been steadily progressing, studies on the frequent occurrence of tail movements every time a piglet is born have not yet been studied [15-18].

The overall objective of this study was to examine the effect of parity on farrowing and weaning traits for sows. Hence, this study was conducted to investigate the relationship between farrowing traits, and birth interval and tail movement according to sow breeding, and to utilize the basic data obtained in sow farm management.

\section{Materials and Methods}

\section{Animals and housing animal}

The experimental period was from May 2017 to May 2018 at a farm in Hadong Country. Twenty Yorkshire $\times$ Landrace F2 crossbred sows were used. The sows used in this study were between the first and sixth parity in gilt (P1), parity $2-5$ (P3), and parity 6-9 (P6), respectively, representing gilt, middle parity, and last parity (parity that is culled from most farms). Sows were housed in individual farrowing pens (W $220 \times \mathrm{D} 180 \times \mathrm{H} 120 \mathrm{~cm}$ ) on a partially slatted plastic floor. To observe behavior, the cameras were installed at 4 sides located $2 \mathrm{~m}$ from the farrowing pens. The pens were equipped with a nipple drinker, feed trough, and lamp heater. The sows were fed twice a day with a standard concentrated diet (3.0 $\mathrm{kg}$ of a diet with $13.2 \mathrm{MJ}$ digestible energy and $131 \mathrm{~g}$ crude protein $(16 \%$ or less $) / \mathrm{kg}$ ). Water was freely available from a drinker within the feed trough. The sows house temperature averaged 20.5 $\pm 2.1^{\circ} \mathrm{C}$.

\section{Observation, data collection during farrowing}

Data collection by parity was classified into three categories:

(1) Reproductive performance (gestation length days, total number of piglets born, number of live born piglets, number of still born piglets, total piglet birth weight);

(2) Weaning traits (weaning period days, number of piglets weaning, total piglet weaning weight, and duration traits);

(3) The duration traits examined were: farrowing duration (the time interval between the birth of the first and last piglets), placenta expulsion duration (the time interval between the first placenta being visible and the last placenta being expelled), time from last piglet to first placenta (the time of appearance of first placenta relative to the last piglet), average birth interval (the first born piglet was assumed to have a reference point of $0 \mathrm{~min}$ ), and tail wagging behavior (rapid swinging of the tail from side to side and a few seconds before piglet birth tail movement).

Data of reproduction performance and weaning traits were obtained from computerized records, and software programs (PIG PLAN, Ezfarm, Inc., Korea) were used for the management of breeding stock on the experimental farm. The tail wagging behavior of the sows was recorded duringe farrowing from the first to the last piglet. Whenever movement, was observed, the frequency was analyzed.

In principle, no attempts were made to interfere with the natural farrowing of the sows. Furthermore, there was no human intervention to control aggression towards the newborns and no assistance of the piglets to find a teat. No savagers behavior occurred in the sows observed in the experiment (gilts that displayed offspring-directed aggression, i.e., attacked, bit, or even killed offspring) [2].

\section{Statistical analysis}

All data were analyzed with $R$ package ( $R$ version 3.31, $R$ Foundation for Statistical Computing, Tokyo, Japan). Effect of parity on reproduction performance, weaning traits, and duration traits were 
analyzed using ANOVA. The data for farrowing traits and weaning traits were investigated of mean and standard deviation. The data for duration traits of frequency and minute was not normally distributed, based on the Shapiro-Wilk test. For the calculation of significant differences between the days of observation, the Kruskal- Wallist test was used. A $p$-value of $<0.05$ was considered significant. To analyze the relationship between variables in the birth intervals and tail wagging, a Pearson correlation was conducted.

\section{Results and Discussion}

Gestation length was higher in P6 than P1 and P3 in different parity sows (Table 1). The maximum value in $\mathrm{P} 1$ and $\mathrm{P} 3$ was 117 days, but the median value in P6 was 117 days $(p<0.05)$ [12]. Studies have shown that stress during pregnancy is more prevalent in gilts than in other sows owing to limited behavior during gestation, but P6 had the longest gestation length in the present study. This means that the higher the parity, the greater the physical impact of sows than the effect of stress on reproductive performance. It also supports previous research on sows, indicating that stress may delay farrowing and recurrent estrus periods [19].

The total number of piglets born $(p=0.113)$, number of live piglets born $(p=0.118)$, number of still born piglets $(p=0.151)$, and total piglet birth weight $(p=0.117)$ were not affected by parity. The number of live piglets in $\mathrm{P} 1$ was higher than that in the other parities by an average of $15.6 \pm 2.1$. In contrast, the average number of weaning piglets was 10 heads. This indicates that gilts lacked maternal behavior, and weak piglets were likely to starve to death owing to competition [20,21].

In addition, the influence of sow behavior on piglets mortality due to crushing reported [15] found that piglets are most vulnerable to crushing during the first $24 \mathrm{~h}$ of life, when they are spending much of their time near the udder and have relatively poor mobility. Furthermore, there are reported the piglets born to older sows have reliable thermoregulation [27].

It is well recognize that in the sow most stillbirths occur intrapartum as a result of dystocia and the delayed expulsion of the litter [28]. There are substantial data on stillbirth rates in sows [29,30]. The results of this experiment suggest that the number of stillborn is less than 1 in most parities and it is difficult to regard them as dystocia.

Weaning traits of sows also did not significantly differ $(p>0.05)$, but the weaning period from P1 was lower than that in other parities $(p=0.170)$. The results indicated that the gilts reflect piglet relationships, which was consistent with a theoretically predicted model of weaning conflict.

This is similar to previous studies in which there is a difference in the degree of stress and maternal at the farrowing, and a longer weaning period in sows of higher parities than gilts [22].
The number of piglets weaning was 10 on average in both $\mathrm{P} 1$, P3, and P6 $(p<0.05)$. However, the mean the total piglet weaning weight $(p=0.377)$ in $\mathrm{P} 6(62.0 \pm 10.4 \mathrm{~kg})$ was higher than that in $\mathrm{P} 1(54.9 \pm 10.2 \mathrm{~kg})$ and $\mathrm{P} 3(58.4 \pm 13.6 \mathrm{~kg})$. This indicated that the number of piglets weaning was the same, but the different parities of the sow and her piglets could influence their interactions with one another [23].

The farrowing duration was the lowest at $22.2 \mathrm{~min}$ in P6 and, but the max value was $42.2 \mathrm{~min}$ more than the other parities $(p=$ $0.355)$. The placenta expulsion duration was higher in $\mathrm{P} 6$ than in P1 and P3 $(p=0.447)$. The time from last piglet to first placenta was lower in $\mathrm{P} 3$ than that in the other parities $(p=0.206)$. The average birth interval was be higher in $\mathrm{P} 3$ than that in the other parities $(p=0.156)$.

The tail wagging (count) behavior was higher in P6 than that in the other parities $(p=0.065)$. There were no significant differences between parities on the placenta expulsion duration, time from last piglet to first placenta, birth intervals, as well as tail wagging $(p>0.05)$. Fig. 1 shows of the birth interval of piglets compared to parity. There was no significant difference $(p>0.05)$, but the distributions of graphs were larger in P1 and P3 than that in P6. This was supported by the fact that $\mathrm{P} 1$ was a gilts group with a large distribution, whereas in $\mathrm{P} 3$ the maximum number of stillborns was 4 heads (Table 1). [4] reported that the averages decreased with birth interval with an increase in birth order. However, in a study of gilts, the average of birth interval decreased with an increase of birth order, which was inconsistent with the results from the present experiment [2].

Fig. 2 shows a comparison between the frequency of tail wagging behavior and parities of sows. P1, P3, and P6 showed an increase in tail wagging with the first-born piglet, but this decreased considerably when the last piglet was born $(p>0.05)$. Table 2 shows the correlation between the birth interval of the piglets and tail waggling behavior of sows. This showed the same negative correlation in all parities. In P6, the slope was -0.832 , indicating a significantly negative correlation $(p<0.001)$. In the present experiment, the frequency of tail movement in sow behavior was active, and the piglets born last were healthy and were not still born. esearch reported that the active movement of the tail of pigs was previously studied as an indicator of positive emotions, but the results from the present study were contradictory [11]. We suggested that tail wagging is one of the more important parts of sow pain. In the case of piglets that tail wagging increased significantly in a food frustration situation [24]. Increased amounts of tail wagging were also found after surgical procedures like tail docking [25] and castration [26]. These results are in agreement with suggested that skin irritation (which is likely for pigs with tail damage) would induce tail wagging. 
Table 1. Descriptive statistics of the Yorkshire $\times$ Landrace F2 crossbred P1, P3, and P6

\begin{tabular}{|c|c|c|c|c|c|c|}
\hline & Parity & Avg SD & Med & Min & Max & $p$-value \\
\hline \multicolumn{7}{|l|}{ Sows traits } \\
\hline \multirow[t]{3}{*}{ Gestation length (days) } & $\mathrm{P} 1$ & $115.4 \pm 1.3$ & 116 & 114 & 117 & $<0.05$ \\
\hline & P3 & $115.5 \pm 1.2$ & 115 & 114 & 117 & \\
\hline & P6 & $116.7 \pm 0.9$ & 117 & 115 & 118 & \\
\hline \multirow[t]{3}{*}{ Total number of piglets born (heads) } & $\mathrm{P} 1$ & $16.8 \pm 1.8$ & 17 & 14 & 19 & 0.113 \\
\hline & P3 & $15.5 \pm 3.1$ & 15 & 12 & 21 & \\
\hline & P6 & $12.4 \pm 3.2$ & 12 & 7 & 17 & \\
\hline \multirow[t]{3}{*}{ Number of piglets born live (heads) } & $\mathrm{P} 1$ & $15.6 \pm 2.1$ & 16 & 13 & 18 & 0.118 \\
\hline & P3 & $14.0 \pm 3.2$ & 13 & 10 & 19 & \\
\hline & P6 & $11.0 \pm 3.4$ & 11 & 6 & 16 & \\
\hline \multirow[t]{3}{*}{ Number of piglets still born (heads) } & $\mathrm{P} 1$ & $0.2 \pm 0.5$ & 0 & 0 & 1 & 0.151 \\
\hline & P3 & $1.3 \pm 1.5$ & 1 & 0 & 4 & \\
\hline & P6 & $1.1 \pm 0.3$ & 0 & 0 & 1 & \\
\hline \multirow[t]{3}{*}{ Total piglet birth weight (kg) } & $\mathrm{P} 1$ & $16.8 \pm 2.1$ & 16 & 14 & 20 & 0.117 \\
\hline & P3 & $14.7 \pm 3.2$ & 14 & 10 & 19 & \\
\hline & P6 & $12.4 \pm 3.5$ & 12 & 7 & 18 & \\
\hline \multicolumn{7}{|l|}{ Weaning traits } \\
\hline \multirow[t]{3}{*}{ Weaning period (days) } & $\mathrm{P} 1$ & $23.0 \pm 7.8$ & 24 & 13 & 34 & 0.170 \\
\hline & P3 & $20.7 \pm 2.7$ & 19 & 18 & 24 & \\
\hline & P6 & $20.2 \pm 4.8$ & 19 & 16 & 32 & \\
\hline \multirow[t]{3}{*}{ Weaning piglets (heads) } & $\mathrm{P} 1$ & $10.4 \pm 1.5$ & 11 & 8 & 12 & $<0.05$ \\
\hline & P3 & $10.0 \pm 0.6$ & 10 & 9 & 11 & \\
\hline & P6 & $10.4 \pm 1.7$ & 11 & 8 & 12 & \\
\hline \multirow[t]{3}{*}{ Total piglets weaning weight $(\mathrm{kg})$} & $\mathrm{P} 1$ & $54.9 \pm 10.2$ & 56 & 38 & 66 & 0.377 \\
\hline & P3 & $58.4 \pm 13.6$ & 59 & 43 & 77 & \\
\hline & P6 & $62.0 \pm 10.4$ & 58 & 54 & 81 & \\
\hline \multicolumn{7}{|l|}{ Durations traits } \\
\hline \multirow[t]{3}{*}{ Duration of farrowing (min) } & $\mathrm{P} 1$ & $25.9 \pm 6.1$ & 26 & 18 & 33 & 0.355 \\
\hline & P3 & $29.5 \pm 3.8$ & 29 & 25 & 33 & \\
\hline & P6 & $22.2 \pm 13.3$ & 21 & 5 & 42 & \\
\hline \multirow[t]{3}{*}{ Duration of placenta expulsion (min) } & $\mathrm{P} 1$ & $141.0 \pm 263.0$ & 15 & 0 & 536 & 0.447 \\
\hline & P3 & $192.0 \pm 423.0$ & 17 & 0 & 1,056 & \\
\hline & P6 & $663.0 \pm 1,802.0$ & 23 & 0 & 5464 & \\
\hline \multirow[t]{3}{*}{ Time from last piglet to first placenta (min) } & $\mathrm{P} 1$ & $74.0 \pm 117.0$ & 25 & 0 & 248 & 0.206 \\
\hline & P3 & $34.0 \pm 37.0$ & 31 & 0 & 80 & \\
\hline & P6 & $87.0 \pm 79.0$ & 83 & 1 & 248 & \\
\hline \multirow[t]{3}{*}{ Average birth interval (min) } & $\mathrm{P} 1$ & $125.6 \pm 94.8$ & 114 & 0 & 357 & 0.156 \\
\hline & P3 & $229.3 \pm 125.0$ & 229 & 0 & 455 & \\
\hline & P6 & $167.1 \pm 92.0$ & 189 & 0 & 340 & \\
\hline \multirow[t]{3}{*}{ Behavior of tail wagging (count) } & $\mathrm{P} 1$ & $5.3 \pm 4.3$ & 4 & 1 & 16 & 0.065 \\
\hline & P3 & $5.6 \pm 2.3$ & 4 & 2 & 9 & \\
\hline & P6 & $7.8 \pm 6.7$ & 5 & 0 & 24 & \\
\hline
\end{tabular}

Twenty sows used in this study were between the first and sixth parity in gilts (P1), parity 2-5 (P3), and parity 6-9 (P6).

Avg, average; SD, standard deviation; Med, median; Min, minimum; Max, maximum. 
In conclusion, the data showed that the reproduction performance, total number of piglets born and piglet weight were higher in the gilts than others groups, and the weaning trait was highest in the P6 group. This study examined the relationship between reproductive performance, birth interval and tail motion according to sow parity. Regarding the duration trait, farrowing duration was lower in P6 than other parities, but placenta expulsion duration and tail wagging were higher in $\mathrm{P} 6$ than that in the other parties. In contrast the gilts housing environment changes, they might be stressful and became more anxious and uneasy, resulting in increased standing behavior. Based on the results of this study, we believed that effect of different parties on reproductive performance and tail behavior in sow. This can help producers to take ap-
Table 2. Correlation between the birth interval of the piglets and the tail waggling behavior of sows

\begin{tabular}{lcc}
\hline Parity & $\begin{array}{c}\text { Birth interval: } \\
\text { tail wagging correlation }\end{array}$ & $\boldsymbol{p}$-value \\
\hline P1 & -0.441 & 0.087 \\
P3 & -0.428 & 0.097 \\
P6 & -0.832 & 0.001 \\
\hline
\end{tabular}

Twenty sows used in this study were between the first and sixth parity in gilts (P1), parity 2-5 (P3), and parity 6-9 (P6).

propriate measures to prevent further of the problem as basic data in sow farm management. Through further experiments involving correlations between dystocia and tail movement of sows, a behavioral study of different parties to dystocia is also needed.

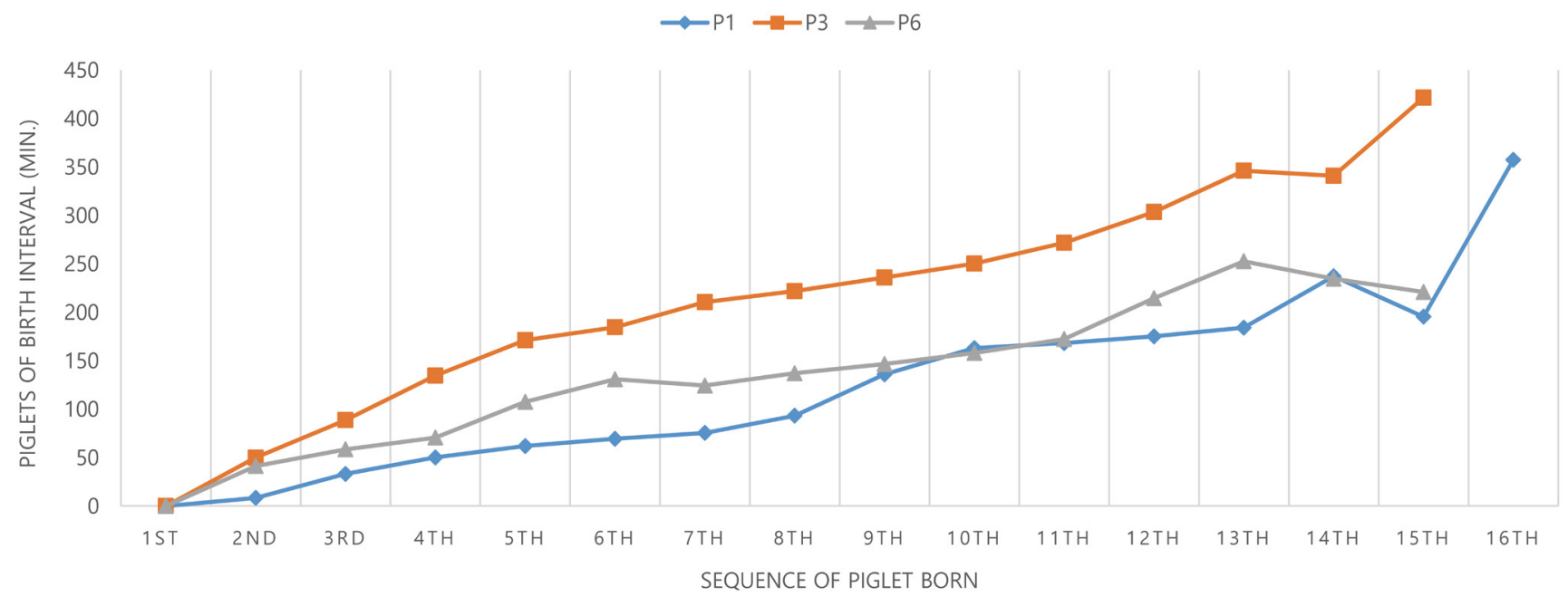

Fig. 1. Comparison of the intervals of birth in piglets. Twenty sows used in this study were between the first and sixth parity in gilts (P1), parity $2-5$ (P3), and parity 6-9 (P6).

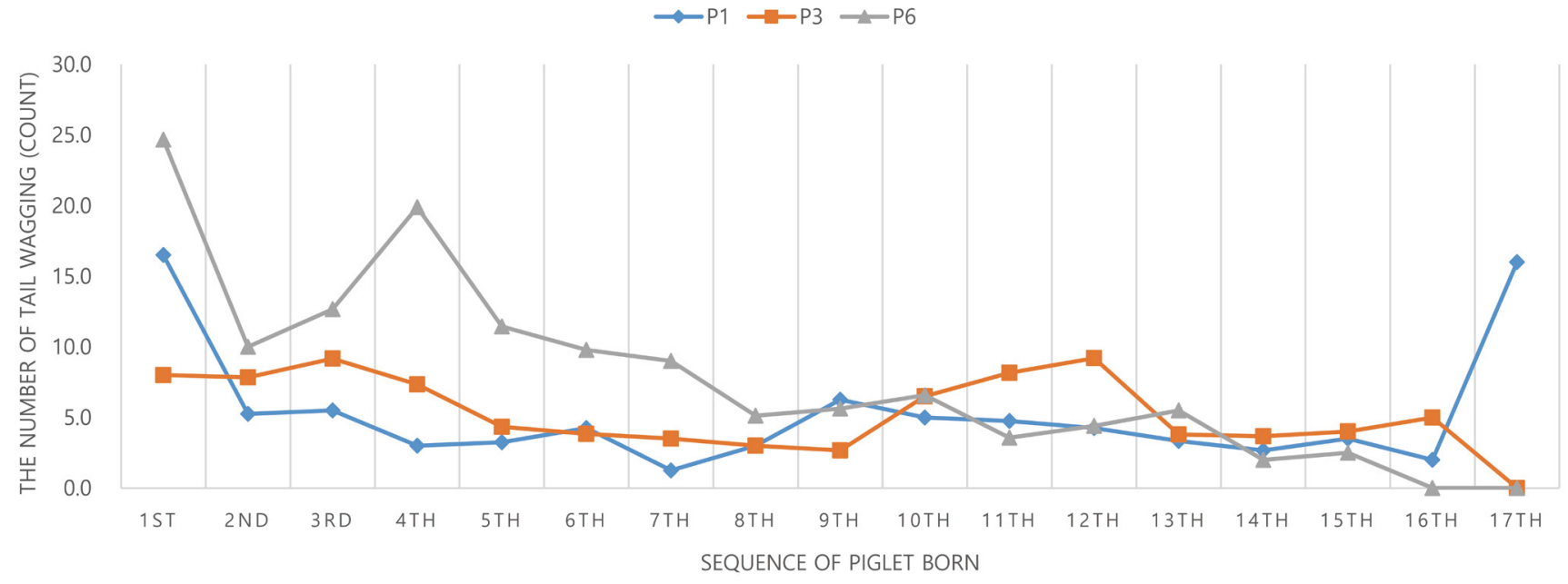

Fig. 2. Comparison of the frequency of tail wagging behavior effect to the parity of sows. Twenty sows used in this study were between the first and sixth parity in gilts (P1), parity 2-5 (P3), and parity 6-9 (P6). 
JAST

\section{Competing interests}

No potential conflict of interest relevant to this article was reported.

\section{Funding sources}

This work was carried out with the support of "Cooperative Research Program for Agriculture Science \& Technology Development (Project No.PJ-01278101 )" Rural Development Administration, Korea.

\section{Acknowledgements}

This research was supported by the "RDA research Associate Fellowship Program (2019)" of the National Institute of Animal Science, Rural Development Administration, Korea.

\section{Availability of data and material}

Upon reasonable request, the datasets of this study can be available from the corresponding author.

\section{Authors' contributions}

Conceptualization: Lee JY.

Data curation: Yang KY.

Formal analysis: Jeon JH.

Methodology: Yang KY,Jeon JH.

Software: Kim JB, Kwon KS.

Vlidation: Choi HC.

Investigation: Lee JY.

Writing-original draft: Yang KY.

Writing-review \& editing: Lee JY, Choi HC, Jeon JH.

\section{Ethics approval and consent to participate}

Protocols involving the use of experimental animals were conducted in accordance the ethical and scientific guidelines of the animal Experiment Ethics Committee of RDA (No: NIAS-2017064).

\section{ORCID}

Ka Young Yang

Jung Hwan Jeon

Hee Chul Choi

Jong Bok Kim

Jun Yeob Lee
Kyeong Seok Kwon https://orcid.org/0000-0003-3232-6075

https://orcid.org/0000-0001-9725-547X

https://orcid.org/0000-0001-5656-3441

https://orcid.org/0000-0002-8132-0983

https://orcid.org/0000-0001-7609-4208

https://orcid.org/0000-0001-8074-586X

\section{References}

1. Yang KY, Jeon JH, Kwon KS, Choi HC, Ha JJ, Kim JB, et al. Classification of behavior at the signs of parturition of sows by image information analysis. J Korea Acad Ind Soc. 2018;19:607-13.
2. van Rens BT, van der Lende T. Parturition in gilts: duration of farrowing, birth intervals and placenta expulsion in relation to maternal, piglet and placental traits. Theriogenology. 2004; 62:331-52.

3. Wulbers-Mindermann M, Algers B, Berg C, Lundeheim N, Sigvardsson J. Primiparous and multiparous maternal ability in sows in relation to indoor and outdoor farrowing systems. Livest Prod Sci. 2002;73:285-97.

4. Pedersen LJ, Damm BI, Marchant-Forde JN, Jensen KH. Effects of feed-back from the nest on maternal responsiveness and postural changes in primiparous sows during the first $24 \mathrm{~h}$ after farrowing onset. Appl Anim Behav Sci. 2003;83:109-24.

5. Mota-Rojas D, Martinez-Burnes J, Trujillo-Ortega ME, Alonso-Spilsbury ML, Ramirez-Necoechea R, Lopez A. Effect of oxytocin treatment in sows on umbilical cord morphology, meconium staining, and neonatal mortality of piglets. Am J Vet Res. 2002;63:1571-4.

6. Leenhouwers JI, de Almeida Junior CA, Knol EF, van der Lende T. Progress of farrowing and early postnatal pig behavior in relation to genetic merit for pig survival.J Anim Sci. 2001;79:1416-22.

7. Vallet JL, Freking BA, Miles JR. Effect of empty uterine space on birth intervals and fetal and placental development in pigs. Anim Reprod Sci. 2011;125:158-64.

8. Knox RV. Physiology and endocrinology symposium: factors influencing follicle development in gilts and sows and management strategies used to regulate growth for control of estrus and ovulation.J Anim Sci. 2019;97:1433-45.

9. Viscardi AV, Hunniford M, Lawlis P, Leach M, Turner PV. Development of a piglet grimace scale to evaluate piglet pain using facial expressions following castration and tail docking: a pilot study. Front Vet Sci. 2017;4:51.

10. Dalla Costa E, Minero M, Lebelt D, Stucke D, Canali E, Leach MC. Development of the horse grimace scale (HGS) as a pain assessment tool in horses undergoing routine castration. PLOS One. 2014;9:e92281.

11. Reimert I, Bolhuis JE, Kemp B, Rodenburg TB. Indicators of positive and negative emotions and emotional contagion in pig. Physiol Behav. 2013;109:42-50.

12. Kim DH, Park JY, Woo JH. Effects of gestation housing and parity on the farrowing performance and behaviour of sows during the pregnancy, farrowing and lactation. J Anim Sci Technol. 2003;45:1067-78.

13. Dailey JW, McGlone JJ. Pregnant gilt behavior in outdoor and indoor intensive pork production systems. Appl Anim Behav Sci. 1997;52:45-52.

14. McGlone JJ, Fullwood SD. Behavior, reproduction, and immunity of crated pregnant gilts: effects of high dietary fiber 
and rearing environment. J Anim Sci. 2001;79:1466-74.

15. Marchant JN, Broom DM, Corning S. The influence of sow behaviour on piglet mortality due to crushing in an open farrowing system. Anim Sci. 2001;72:19-28.

16. Chidgey KL, Morel PCH, Stafford KJ, Barugh IW. Observations of sows and piglets housed in farrowing pens with temporary crating or farrowing crates on a commercial farm. Appl Anim Behav Sci. 2016;176:12-8.

17. Goumon S, Leszkowova I, Simeckova M, Illmann G. Sow stress levels and behavior and piglet performances in farrowing crates and farrowing pens with temporary crating. J Anim Sci. 2018; 96:4571-78.

18. Carter SD, Hill GM, Mahan DC, Nelssen JL, Richert BT, Shurson GC. Effects of dietary valine concentration on lactational performance of sows nursing large litters. J Anim Sci. 2000; 78:2879-84.

19. Estienne MJ, Harper AF. Type of accommodation during gestation affects growth performance and reproductive characteristics of gilt offspring. J Anim Sci. 2010;88:400-7.

20. Arey DS, Sancha ES. Behaviour and productivity of sows and piglets in a family system and in farrowing crates. Appl Anim Behav Sci. 1996;50:135-45.

21. Cronin GM, Simpson GJ, Hemsworth PH. The effects of the gestation and farrowing environments on sow and piglet behaviour and piglet survival and growth in early lactation. Appl Anim Behav Sci. 1996;46:175-92.

22. Puppe B, Tuchscherer A. The development of suckling frequency in pigs from birth to weaning of their piglets: a sociobiological approach. Anim Sci. 2000;71:273-9.

23. Chidgey KL, Morel PCH, Stafford KJ, Barugh IW. Sow and piglet behavioral associations in farrowing pens with temporary crating and in farrowing crates. J Vet Behav. 2017;20:91101.

24. Zonderland JJ, van Riel JW, Bracke MBM, Kemp B, den Hartog LA, Spoolder HAM. Tail posture predicts tail damage among weaned piglets. Appl Anim Behav Sci. 2009;121:16570.

25. Noonan GJ, Rand JS, Priest J, Ainscow J, Blackshaw JK. Behavioural observations of piglets undergoing tail docking, teeth clipping and ear notching. Appl Anim Behav Sci. 1994;39:203-13.

26. Hay M, Vulin A, Genin S, Sales P, Prunier A. Assessment of pain induced by castration in piglets: behavioral and physiological responses over the subsequent 5 days. Appl Anim Behav Sci. 2003;82:201-8.

27. Santiago PR, Martinez-Burnes J, Mayagoitia AL, RamirezNecoechea R, Mota-Rojas D. Relationship of vitality and weight with the temperature of newborn piglets born to sows of different parity. Livest Sci.2019;220:26-31.

28. Noakes D. Dystocia and other disorders associated with parturition. In: Noakes DE, Parkinson TJ, England GCW, editors. Veterinary Reproduction and Obstetrics. 2009. p. 207-305.

29. Vincze B, Gaspardy A, Kezer FL, Palffy M, Bangha Z, Szenci $\mathrm{O}$, et al. Fetal metacarpal/metatarsal bone thickness as possible predictor of dystocia in Holstein cows. J Dairy Sci. 2018;101:10283-9.

30. Okinda C, Lu M, Nyalala I, Li J, Shen M. Asphyxia occurrence detection in sows during the farrowing phase by inter-birth interval evaluation. Comput Electron Agric. 2018;152:221-32. 